\title{
Positive effect of heterogeneity of difference on the same-different disparity in letter matching
}

\author{
LESTER E. KRUEGER \\ Ohio State University, Columbus, Ohio
}

\begin{abstract}
Subjects judged whether two adjacent letters were identical or different. Different pairs were similar, for example HM, DO, VY, or dissimilar, for example DY, HV, MO. According to the noisyoperator theory, increasing the heterogeneity of difference (external noise) by intermixing similar and dissimilar different pairs ought to produce faster but less accurate responses on different trials. As predicted, the tendency to make more false-different responses (i.e., errors on same pairs) decreased when the similar and dissimilar different pairs were intermixed rather than presented in separate blocks. The fast-same effect did not change, however, seemingly due to criterion misadjustment. Proctor and Rao apparently found no effect of heterogeneity of difference on RT and errors because they used a less sensitive procedure (between-subjects design; different set of letters for more and less heterogeneous conditions). Consistent with the internal-noise principle, but not with the response-competition model of Eriksen, O'Hara, and Eriksen, the enhanced fastsame effect on blocks containing only similar (vs. dissimilar) different pairs was accompanied by an increased preponderance of false-different errors.
\end{abstract}

When people judge whether two letters are identical or not, same judgments typically are faster than different judgments. According to the noisy-operator theory (Krueger, 1978), internal noise more often changes physical matches into spurious perceived mismatches than vice versa. As a result, different judgments are slower, owing to more rechecking of perceived mismatches, and subjects typically make more false-different responses (i.e., errors on same trials) than false-same ones.

External noise also must be considered, however. Variability in the perceived difference count is the joint effect of variability within the subject (internal noise) and variability dependent on the investigator (external noise), who controls the number of differing features present (Krueger, 1978, 1979). While internal noise ought to produce faster but less accurate responses on same trials, external noise ought to produce faster but less accurate responses on different trials. When the different pairs vary in the degree of their featural overlap with the same pairs, then the similar different pairs would tend to be confused with same pairs and produce false-same errors, and the rechecking needed to eliminate such errors would slow down the same judgments. In effect, the similar different pairs would undermine the same distribution on the perceived difference count.

Typically, responses are faster but less accurate on same pairs in letter matching, which indicates that internal noise predominates. Krueger and Shapiro (1981) concluded that "external noise is largely absent in single-letter match-

The author is grateful to Robert W. Proctor for helpful comments on an earlier version of this report. Requests for reprints should be sent to Lester E. Krueger, Human Performance Center, Ohio State University, 404-B W. 17th Avenue, Columbus, OH 43210. ing.... English letters apparently have evolved so as to be nearly equally discriminable from each other, especially uppercase letters, which are the mainstay of lettermatching studies. Krueger (1978) fitted the noisy-operator theory satisfactorily to various data using the assumption of uniform discriminability of English letters"' (p. 575). However, Proctor and Rao (1983b) disputed Krueger's assumption "that external noise is not a significant factor" (p. 27) in letter matching. They found considerable variability in letter differences in Krueger's (1978) letter sets, and concluded that "because the internal noise principle relies on the assumption of homogeneity of difference to predict the same-different disparity and this assumption is not even approximately correct, internal noise is most likely not the basis for the same-different disparity" (p. 29).

However, Proctor and Rao presented no quantitative analyses to show that sufficient external noise was present to neutralize the effect of internal noise, and their concern that the presence of external noise "seems to be fatal to the internal noise principle"' (p. 23) would seem to have been laid to rest when they themselves (Proctor \& Rao, 1983a, Experiment 3) subsequently tested the external-noise principle. Using various confusion matrices and distinctive feature systems, they devised two letter sets which differed in the heterogeneity of difference within the set. In their own data, they confirmed that different $\mathrm{RT}$ s on pairs from the set A, E, N, T (homogeneous set) varied less than those on pairs from the set $E$, P, R, W (heterogeneous set). Letter set was a betweensubjects variable; 12 subjects received same and different pairs constructed from the homogeneous set, and another 12 received pairs constructed from the heterogeneous set. Proctor and Rao found slight and insignificant 
effects in the predicted direction: subjects receiving the heterogeneous set were $8 \mathrm{msec}$ lower on the fast-same effect and $0.1 \%$ lower on the preponderance of falsedifferent errors. Their null finding is consistent with the view that letters, for all their differences, are remarkably similar or alike in many or most respects (Eriksen, O'Hara, \& Eriksen, 1982). Quite likely, they would have found a definite effect had they used materials permitting greater heterogeneity of difference. Krueger and Shapiro (1985) tested pairs of lines, whose difference ratio on length varied fourfold (i.e., from $1: 2$ to $1: 8$ ), and they found an effect in the predicted direction on both errors and RT, although only the error effect was significant. When the difference ratio was varied rather than kept constant for the subject, the error disparity shifted toward false-same responses by $2 \%$, whereas the fast-same effect decreased by $7 \mathrm{msec}$.

One ought not to be too hasty, however, in dismissing completely the external-noise factor in letter matching. Even if external noise is not all-powerful and rarely overwhelms the internal noise present, it ought nonetheless to have at least some effect. Proctor and Rao may have erred in both directions, first in overstating the role of external noise in letter matching, and then in understating it. The true assessment may lie somewhere in between. The present study was undertaken to test whether certain factors in Proctor and Rao's (1983a) experimental design prevented them from obtaining a positive effect.

Both of the previous tests of the external-noise principle (Krueger \& Shapiro, 1985; Proctor \& Rao, 1983a) used between-subjects experimental designs. If more sensitive, within-subjects designs had been used instead, as will be done here, more reliable effects on both RT and errors might have been found. In addition, the use of different letters in Proctor and Rao's two letter sets may have introduced extraneous, confounding factors. Responses tended to be slower, but more accurate, with their heterogeneous letter set, so it is not clear which letter set was more difficult to process. An increased level of internal noise on the heterogeneous set, if present, would have tended to counteract the RT and error effects of its increased level of external noise or heterogeneity of difference, and this might explain their null effect. Furthermore, in the heterogeneous set, only one of the six different pairs (i.e., PR) differed considerably in RT from the other pairs, whose variability on RT was about the same as that of the homogeneous set.

In the present test, a within-subjects design was used, and the same letter pairs were used in the low- and highexternal-noise conditions, which not only removed possible confounding factors, but also ensured that half of the different pairs differed considerably in RT from the other half. The same six letters (D, H, M, O, V, Y) that Chignell and Krueger (1984) used in their Experiment 2 were used here. Chignell and Krueger had found that the dissimilar different pairs they used (HV, OM, DY) were responded to about $50 \mathrm{msec}$ faster and $2 \%$ more accurately than were the similar pairs (HM, OD, VY). They intermixed the similar and dissimilar different pairs, but never tested them in separate blocks, which was done here, using all six dissimilar and six similar pairs, that is, both orders of each pair. If letters differ sufficiently in their discriminability from each other, and if the external-noise principle is correct, then, it was felt, the present test ought to be sensitive enough to detect the predicted effect of increased external noise, that is, faster but less accurate responses on different trials.

\section{Response-Competition Principle}

Chignell and Krueger found that, with simultaneous presentation, same and dissimilar different pairs were responded to equally fast, whereas false-same errors as well as RT were much higher on similar than on dissimilar different pairs. These results support the Eriksen et al. (1982) response-competition model, which attributes slower different judgments to matching features on similar different pairs. Chignell and Krueger said that the response-competition model must be supplemented by the internal-noise principle, however, because false-different errors on same pairs consistently exceeded even falsesame errors on similar different pairs.

A second objective of the present study was to determine whether the same evidence supporting the responsecompetition model would be found when similar and dissimilar different pairs were presented in separate blocks of trials. By intermixing the two types of different pairs, Chignell and Krueger forced their subjects to use a common decision criterion (Krueger, 1985), irrespective of different type. The evidence supporting the responsecompetition model (equally fast RT on same and dissimilar different pairs; smaller preponderance of false-different errors with similar than with dissimilar different pairs) may have resulted because the criterion for responding versus rechecking was set too low on the perceived difference count relative to the dissimilar different pairs and too high relative to the similar different pairs. According to the internal-noise principle, the fast-same effect ought to be smaller, but still be present, on pure blocks with dissimilar (vs. similar) different pairs, and the preponderance of false-different errors ought to be larger, not smaller, on pure blocks with similar (vs. dissimilar) different pairs. In previous studies, errors increased more on same trials under difficult conditions (e.g., Krueger \& Gott, 1985; Krueger \& Shapiro, 1985), and increased similarity on different trials may simply be one more way in which task difficulty may be increased.

\section{EXPERIMENTS 1 AND 2}

The present two experiments were identical, except that, to remove all external noise from the pure block, each subblock of a pure block in Experiment 2 was restricted to pairs formed from just two of the six letters. Experiment 1 found the predicted effect of external noise on errors, but not RT, and it seemed likely that the residual external noise present on the pure block, owing to varia- 
tion in difficulty among similar or dissimilar different pairs, had attenuated the effect of pure versus intermixed blocks. In Experiment 1, mean RTs on the six different pairs used ranged from 584 to $616 \mathrm{msec}$ in the pure similar blocks and from 506 to $542 \mathrm{msec}$ in the pure dissimilar blocks. However, the results of Experiment 2 were nearly identical to those of Experiment 1, indicating that withinset heterogeneity had relatively little effect, and therefore the results from the two experiments will be considered together.

\section{Method}

Subjects. Twenty Ohio State University undergraduates participated in Experiment 1 and 20 participated in Experiment 2 in order to receive credit in an introductory psychology course. Data from 6 additional subjects were discarded, 3 because of equipment failure and 3 for exceeding an $8 \%$ error criterion. All subjects had at least $20 / 30$ vision (corrected), as tested with a Snellen chart.

Apparatus. Uppercase letters were presented at a $60-\mathrm{Hz}$ refresh rate and $32-\mathrm{cd} / \mathrm{m}^{2}$ intensity on a greenish-tint, fast-decay P31 phosphor (decay to $1 \%$ intensity at $.25 \mathrm{msec}$ after display offset) by an Imlac PDS-4 graphics computer, which also measured RT to an accuracy of $1 \mathrm{msec}$. The letters, presented as thin, illuminated lines on a dark screen, were software generated, using short line vectors, so as to resemble normal English uppercase letters. Each subject sat alone in a dark room, with the head held fast in a chinrest located $70 \mathrm{~cm}$ from the display screen.

Stimulus materials. Each letter was $.29 \mathrm{~cm}$ wide and $.43 \mathrm{~cm}$ high, and $.19 \mathrm{~cm}$ separated the two letters. The pair thus was $0.77 \mathrm{~cm}\left(0.63^{\circ}\right)$ wide, and it was centered $0.3 \mathrm{~mm}$ above a small $(1.6 \times 1.6 \mathrm{~mm})$ plus sign, which was the fixation mark at the center of the screen.

Six letters $(\mathrm{D}, \mathrm{H}, \mathrm{M}, \mathrm{O}, \mathrm{V}, \mathrm{Y})$ were used to form 6 same pairs, and 12 different pairs, 6 of which were similar (DO, OD, HM, MH, VY, YV) and 6 of which were dissimilar (DY, YD, HV, VH, MO, OM). Each block of 144 regular trials contained 72 same pairs (12 of each of the 6 same pairs) and 72 different pairs (12 each of the 6 similar or 6 dissimilar different pairs on pure blocks; 6 each of the full set of 12 different pairs on mixed blocks). There were four blocks: one pure similar, one pure dissimilar, and two mixed, which were presented contiguously, either before or after (half of the subjects each) the two pure blocks. Half of the subjects received the pure similar block before the pure dissimilar block, and the other half had the reverse order. Four different randomized sequences of letter pairs and trials were used in each experiment.

Each block consisted of four subblocks of 36 trials each in Experiment 1, and three subblocks of 48 trials each in Experiment 2. In Experiment 2, after the 144 trials in a pure block were randomly assigned to the three subblocks, the six letters were reduced or collapsed to a different pair of two letters in each subblock. For half of the subjects, the order of the pairs used in the three subblocks was HM, DO, VY for the pure similar block, and DY, HV, MO for the pure dissimilar block. The other half of the subjects had the reverse order (i.e., VY, DO, HM; MO, HV, DY). Thus, each subblock in a pure block in Experiment 2 contained four particular letter pairs (e.g., HH, MM, HM, MH for the pair HM), which appeared approximately 12 times each; equal weight was given to each of the four particular letter pairs in computing cell means.

Each subblock was preceded by 4 practice trials, and a practice subblock containing the same letter pairs as the first regular subblock preceded the pure similar block, the pure dissimilar block, and the first of the two contiguous mixed blocks. Thus, there were 19 subblocks, or 760 trials (576 regular, 184 practice) in Experiment 1 and 15 subblocks, or 780 trials (576 regular, 204 practice) in Experiment 2.

Procedure. In each trial, the fixation mark appeared alone for $.6 \mathrm{sec}$, then the letter pair appeared as well until the subject responded. No feedback was given on the accuracy of the response during the .5 -sec interval between trials. There was a 15- to 20-sec interval between subblocks, which allowed the next set of trials to be read in from the host computer.

The subjects were instructed to keep their eyes focused at all times just above the fixation mark, between where the two letters would appear, and to respond as rapidly as possible, but not at the expense of accuracy. For half of the subjects, the "same" response was assigned to the right-hand button, and the "different" response to the left-hand button; the other half had the reverse hand assignment. Trials with an RT greater than $3 \mathrm{sec}$ or less than $200 \mathrm{msec}$ were discarded prior to data analysis. Mean RT was computed for correct trials only.

\section{Results and Discussion}

Mean RT and error data are shown in Figure 1 for Experiment 1 and in Figure 2 for Experiment 2. Table 1 presents the $F$ ratios for the two experiments, considered both separately and combined. For the two experiments combined, RT was lower on same pairs $(p<.001)$ but errors were higher $(p<.001)$, which is consistent with the internal-noise principle. Pure blocks had lower errors than mixed blocks $(p<.01)$. Mean RT was lower $(p<.001)$, as were errors also $(p<.001)$, when the different type was dissimilar rather than similar, especially in Experiment 1 (vs. Experiment 2) on errors $[F(1,38)$ $=4.50, p<.05$ ]. Different type interacted significantly with the same-different and pure-mixed variables, both singly and combined (see Table 1), but these interactions are spurious, because the same RT and errors necessarily were held constant for the similar and dissimilar different conditions on mixed blocks. A truer view of the interaction of different type with same-different will be obtained when pure blocks are examined separately below.

The fast-same effect was virtually identical on pure and mixed blocks, both in Experiment 1 (26 vs. $28 \mathrm{msec}$,

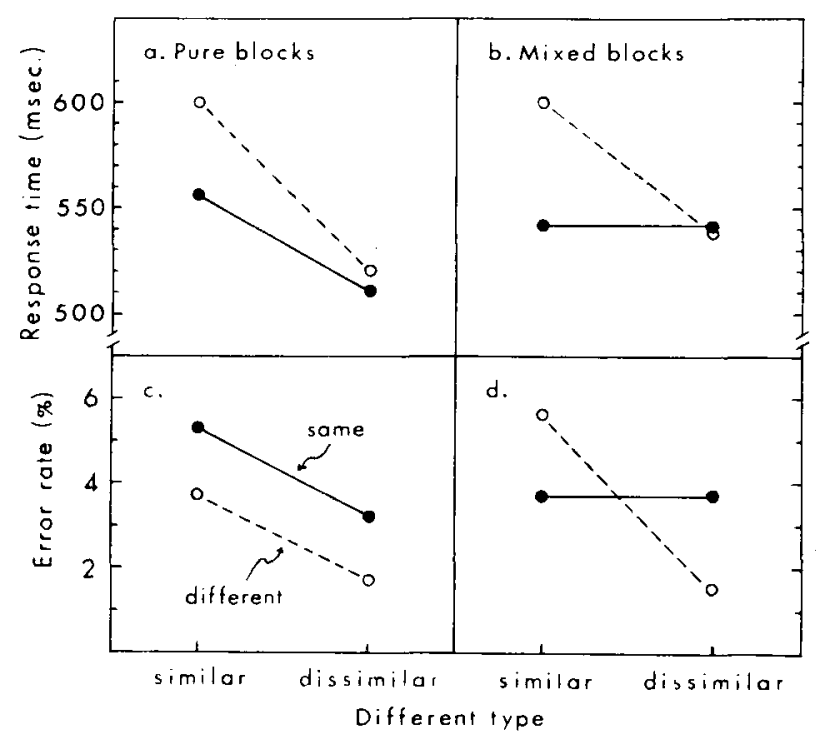

Figure 1. Experiment 1: Mean response time (in milliseconds) and percentage of error rate by trial type (same, similar different, dissimilar different) and pure versus mixed blocks. 


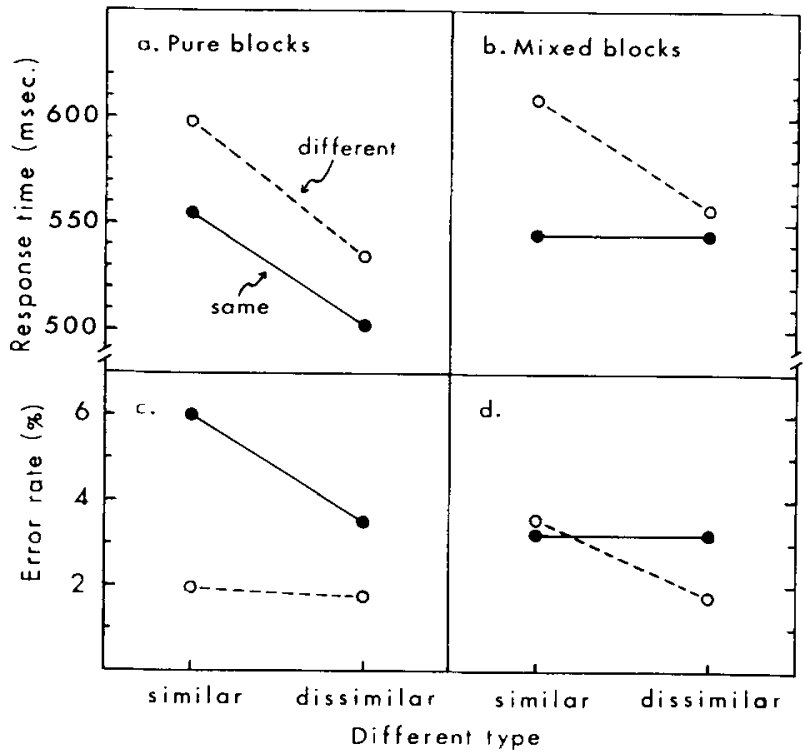

Figure 2. Experiment 2: Mean response time (in milliseconds) and percentage of error rate by trial type (same, similar different, dissimilar different) and pure versus mixed blocks.

$F<1$ ) and in Experiment 2 ( 38 vs. $38 \mathrm{msec}, F<1$ ). By contrast, the preponderance of false-different (vs. falsesame) errors was much larger on pure than on mixed blocks, both in Experiment $1(1.57 \%$ vs. $0.13 \%$, $p<.05)$ and in Experiment $2,(2.91 \%$ vs. $0.56 \%$, $p<.01$ ) (see Figures 1 and 2). Thus, the predicted effect of external noise was found on errors, but not on RT. The constancy of the fast-same effect indicates, however, that the error shift was not due simply to an increased motor bias toward the "same" button on mixed blocks.

Letters thus differ sufficiently in their discriminability from one another so that a more sensitive test (i.e., withinsubjects design; same letter set for pure and mixed blocks) can detect an effect of external noise. The effect of external noise was not sufficient, however, to reverse the preponderance of false-different errors, and it did not affect the fast-same effect at all, so its more modest presence in randomly selected letter pairs need not be as "fatal" to the internal-noise principle as Proctor and Rao (1983b) claimed.

It is not clear why external noise affected only errors. In a line-matching task, Krueger and Shapiro (1985) likewise found a significant effect of external noise on the same-different disparity for errors but not for RT. Perhaps criterion inertia (Krueger, 1985) prevented subjects from properly adjusting both RT and errors to the changed conditions on mixed blocks. The error data suggest that subjects used the same criterion on mixed blocks as on pure dissimilar blocks.

Pure blocks. On pure blocks analyzed separately, the fast-same effect was significant $[F(1,38)=50.75$, $p<.001]$, as was also the preponderance of falsedifferent errors $[F(1,38)=40.97, p<.001]$. Consistent with the internal-noise principle, the latter effects were larger on pure similar (vs. dissimilar) blocks, both on RT $[F(1,38)=14.40, p<.001]$ and errors $[F(1,38)=4.53$, $p<.05]$. Judgments on pure similar (vs. dissimilar) blocks were generally slower $[F(1,38)=104.25$, $p<.001]$ and more errorful $[F(1,38)=28.17$, $p<.001]$, indicating that increased similarity did indeed increase task difficulty.

The same-different disparity was significant on both RT and errors for pure dissimilar blocks analyzed separately as well as for pure similar blocks ( $p<.001$ in all cases). In the mixed condition, by contrast, dissimilar different pairs were as fast as same pairs, which Chignell and Krueger (1984) also found with mixed, simultaneous presentation, presumably due to the presence of similar different pairs.

The larger fast-same effect on pure similar (vs. dissimilar) blocks is consistent with Eriksen et al.'s (1982) response-competition model. They attributed the slow "different" response to competing matching features, which are largely absent on dissimilar different pairs. However, contrary to their model, a fast-same effect nevertheless was found on pure dissimilar blocks, and also the preponderance of false-different errors increased rather than decreased on pure similar (vs. dissimilar) blocks. The present results did not rule out response competition as a factor, but indicate that whatever effect it had was overridden by that of internal noise, which predominated in the present situation.

Table 1

Analysis of Variance of Mean Response Time (RT) and Percentage of Error Rate (PE) for Experiments 1, 2, and 1 and 2 Combined

\begin{tabular}{lccc}
\hline & & \multicolumn{2}{c}{$F$ Ratio } \\
\cline { 3 - 4 } \multicolumn{1}{c}{ Source } & $d f$ & RT & PE \\
\hline & Experiment 1 & & \\
Same-different (SD) & 1,19 & $19.27 \ddagger$ & 3.04 \\
Different type (DT) & 1,19 & $108.83 \ddagger$ & $44.29 \ddagger$ \\
Pure-mixed (PM) & 1,19 & 1.48 & 0.20 \\
SD $\times$ DT & 1,19 & $39.95 \ddagger$ & $19.27 \ddagger$ \\
SD $\times$ PM & 1,19 & 0.06 & $5.05 *$ \\
DT $\times$ PM & 1,19 & $9.52 \dagger$ & 0.00 \\
SD $\times$ DT $\times$ PM & 1,19 & $9.64 \ddagger$ & $10.15 \dagger$ \\
& Experiment 2 & & \\
Same-different (SD) & 1,19 & $28.48 \ddagger$ & $28.77 \ddagger$ \\
Different type (DT) & 1,19 & $92.56 \ddagger$ & $13.72 \ddagger$ \\
Pure-mixed (PM) & 1,19 & $6.70 *$ & 2.36 \\
SD $\times$ DT & 1,19 & $41.45 \ddagger$ & 0.24 \\
SD $\times$ PM & 1,19 & 0.00 & $9.07 \dagger$ \\
DT $\times$ PM & 1,19 & $16.75 \ddagger$ & 0.50 \\
SD $\times$ DT $\times$ PM & 1,19 & $23.63 \ddagger$ & $12.43 \dagger$ \\
& Experiments 1 and 2 & & \\
Same-different (SD) & 1,38 & $47.73 \ddagger$ & $19.49 \ddagger$ \\
Different type (DT) & 1,38 & $201.25 \ddagger$ & $53.79 \ddagger$ \\
Pure-mixed (PM) & 1,38 & $7.04 *$ & 0.16 \\
SD $\times$ DT & 1,38 & $77.84 \ddagger$ & $6.13 *$ \\
SD $\times$ PM & 1,38 & 0.04 & $14.09 \ddagger$ \\
DT $\times$ PM & 1,38 & $24.23 \ddagger$ & 0.29 \\
SD $\times$ DT $\times$ PM & 1,38 & $31.81 \ddagger$ & $22.33 \ddagger$ \\
\hline$p<.05$. & &
\end{tabular}

${ }^{*} p<.05 . \quad \dagger p<.01 . \quad \$ p<.001$. 
Mixed blocks. On mixed blocks analyzed separately, the fast-same effect was significant $[F(1,38)=36.29$, $p<.001]$ but the preponderance of false-different errors was not $(F<1)$. The increased external noise on mixed blocks thus largely neutralized the typical same-different error disparity, but not the fast-same effect, owing perhaps to a criterion misadjustment or decision bias toward "same" on mixed (vs. pure) blocks. Criterion misadjustment could have been produced by the immediately preceding trials as well as the block as a whole. Krueger (1985) found a significant shift in the same-different disparity on errors, but not on RT, depending on whether the preceding judgment was easy (foveal presentation) or difficult (parafoveal presentation).

In a second analysis, each trial in the two mixed blocks was categorized as to its current (Trial $N$ ) and preceding (Trial $N-1$ ) type (same, similar different, dissimilar different). Preceding trial type had a significant effect on RT $[F(2,76)=4.10, p<.025]$ but not on errors $(F<1)$, and current and preceding trial type interacted significantly on RT $[F(4,152)=3.61, p<.01]$ and on errors $[F(4,152)=4.28, p<.01]$. As Table 2 shows, RT was lower, but only on same trials, following a same trial, and there was a preponderance of false-same errors if Trial $N-1$ was dissimilar different. A further analysis involved similar versus dissimilar different on Trial $N-1$ and same versus different on Trial $N$. Following a dissimilar (vs. similar) different trial, the fast-same effect increased ( 24 vs. $15 \mathrm{msec}$ ), but not significantly so $[F(1,38)=1.27]$, whereas the preponderance of falsedifferent errors decreased $[-1.52 \%$ vs. $1.18 \% ; F(1,38)$ $=6.57, p<.025]$. Subjects apparently expected an equally substantial difference on subsequent different trials, and adjusted the criterion upward accordingly on the perceived difference count. Following a similar different trial, the reverse would have occurred, although the net bias toward "same" evident in the mixed blocks suggests that a preceding dissimilar different pair had much more impact than a preceding similar different pair.

\section{CONCLUSIONS}

The present results support the external-noise principle of the noisy-operator theory. Using a more sensitive

Table 2

Experiments 1 and 2, Mixed Blocks: Mean Response Time (RT; in Milliseconds) and Percentage of Error (PE) Rate $\times$ Trial $N-1$ Type and Trial $N$ Type

\begin{tabular}{|c|c|c|c|c|c|c|}
\hline \multirow{3}{*}{$\begin{array}{c}\text { Trial } N-1 \\
\text { Type } \\
\end{array}$} & \multicolumn{6}{|c|}{ Trial $N$ Type } \\
\hline & \multicolumn{2}{|c|}{ Same } & \multicolumn{2}{|c|}{$\begin{array}{c}\text { Similar } \\
\text { Different }\end{array}$} & \multicolumn{2}{|c|}{$\begin{array}{c}\text { Dissimilar } \\
\text { Different }\end{array}$} \\
\hline & $\mathrm{RT}$ & $\mathrm{PE}$ & $\overline{\mathrm{RT}}$ & $\overline{\mathrm{PE}}$ & RT & $\mathrm{PE}$ \\
\hline Same & 528 & 3.75 & 602 & 4.81 & 544 & 0.97 \\
\hline $\begin{array}{l}\text { Similar } \\
\text { Different }\end{array}$ & 559 & 3.82 & 600 & 2.88 & 547 & 2.39 \\
\hline $\begin{array}{c}\text { Dissimilar } \\
\text { Different } \\
\end{array}$ & 552 & 2.53 & 604 & 5.92 & 546 & 2.17 \\
\hline
\end{tabular}

experimental procedure (within-subjects design; same set of letters in more and less heterogeneous conditions) than Proctor and Rao's (1983a), the preponderance of false-different errors was eliminated when similar and dissimilar different trials were intermixed, thus increasing the level of external noise or heterogeneity of difference. No RT shift was found, however. The criterion for rechecking is not always properly adjusted when the internal noise level changes (Krueger, 1985) or, apparently, wher the external noise level changes, as the present data indicate, so a given effect might be expressed solely on $\mathrm{R}^{\prime} \mathrm{T}$ in some cases and solely on errors in other cases. As shown on the right sides of Panels $c$ and $d$ in Figures 1 and 2 , errors on the dissimilar portion of the mixed blocks closely matched those on the pure dissimilar block. Therefore, on mixed blocks, the criterion was set too high on the perceived difference count, thus stifling the shift toward faster different judgments, but enhancing the shift toward more false-same errors. If the criterion had been set too low, owing to relatively greater attention or weight having been accorded to the similar different pairs, then the shift on mixed blocks would have been mainly on RT rather than on errors, with a large decrease or even reversal occurring on the fast-same effect. Perhaps the similar different pairs had less influence on mixed blocks because they occurred only half as frequently as the same pairs, which shared with them the lower region of the perceived difference count.

External noise had a discernible effect on letter matching, but it was not robust enough to neutralize the effect of internal noise, and thus is not as "fatal" to the internalnoise principle as Proctor and Rao (1983b) claimed. The fast-same effect persisted on mixed blocks, along with a small, nonsignificant preponderance of false-different errors. Thus, to a first approximation, letters do seem to be nearly uniformly discriminable (Krueger, 1978; Krueger \& Shapiro, 1981). External noise was intentionally maximized in the present study, but most other studies have avoided introducing additional external noise, so it normally should have even less effect. In my own studies, I have typically used the letters F, G, J, K, and L, which are all highly and about equally discriminable from each other.

External noise can produce huge RT effects, on the order of $300 \mathrm{msec}$, in unidimensional tone comparisons (Krueger, 1979). With tones, the second stimulus may be higher or lower in frequency than the first stimulus, and thus the same distribution may be undermined from both directions by the different distribution (i.e., from negative and positive perceived difference counts). With letters, both portions of the different distribution (similar, dissimilar different pairs) lie on the same (i.e., upper) side of the same distribution and may be quite close together, thus introducing relatively little external noise on mixed blocks, as the present results indicate.

Using mixed blocks and the same letter set as that used here, Chignell and Krueger (1984) found not only a significant fast-same effect, but also a significant prepon- 
derance of false-different errors, contrary to the present results. Their subjects dealt with a variety of horizontal letter separations, letter sizes, and pair durations, however, which presumably made processing more difficult and thus increased the effect of internal noise.

A second objective of the present study was to determine whether the same evidence in favor of Eriksen et al.'s response-competition model would be found here, using pure blocks, as Chignell and Krueger (1984) found, using mixed blocks. The present results with pure blocks proved to be consistent with the internal-noise principle, but not with the response-competition model. First, there was a significant fast-same effect even on pure dissimilar blocks, whose different pairs contained few matching features that could produce response competition. Second, the larger fast-same effect on pure similar (vs. dissimilar) blocks was accompanied by an increased, not decreased, preponderance of false-different errors. Thus, there was no discernible effect of response competition, and increased similarity seems mainly to be one more way to increase task difficulty, which, as previous studies showed, results in a greater increase in errors on same than on different trials (e.g., Krueger \& Gott, 1985; Krueger \& Shapiro, 1985). It should be noted, though, that the latter results on task difficulty, like the present ones, pertain only to late processing. In early processing, letters apparently tend to look very much alike, since, "on the average, the percept will need to develop rather fully before a difference is detected" (Eriksen et al., p. 263). Under high speed stress, the missing-feature principle, not the internal-noise principle, predominates (Krueger \& Chignell, 1985), and the pure blocks containing simi- lar different pairs would have produced a greater number of false-same, not false-different, errors.

\section{REFERENCES}

Chignell, M. H., \& Krueger, L. E. (1984). Further evidence for priming in perceptual matching: Temporal, not spatial, separation enhances the fast-same effect. Perception \& Psychophysics, 36, 257-265.

ERiksen, C. W., O'Hara, W. P., \& Eriksen, B. A. (1982). Response competition effects in same-different judgments. Perception \& Psychophysics, 32, 261-270.

KRUeger, L. E. (1978). A theory of perceptual matching. Psychological Review, 85, 278-304.

KRUEGER, L. E. (1979). A model of unidimensional perceptual matching. Journal of Experimental Psychology: Human Perception \& Performance, 5, 277-288.

KRUEGER, L. E. (1985). Effect of intermixed foveal and parafoveal presentation on same-different judgments: Evidence for a criterioninertia model. Perception \& Psychophysics, 37, 266-271.

Krueger, L. E., \& Chignell, M. H. (1985). Same-different judgments under high speed stress: Missing-feature principle predominates in early processing. Perception \& Psychophysics, 38, 188-193.

Krueger, L. E., \& GotT, R. E. (1985). Effect of lateral masking and letter reversal on same-different judgments. Bulletin of the Psychonomic Society, 23, 185-188.

Krueger, L. E., \& Shapiro, R. G. (1981). A reformulation of Proctor's unified theory for matching-task phenomena. Psychological Review, 88, 573-581.

KRUEGer, L. E., \& Shapiro, R. G. (1985). Effect of interstimulus interval and heterogeneity of difference on same-different judgments of visual patterns. Bulletin of the Psychonomic Society, 23, 43-46.

Proctor, R. W., \& RAo, K. V. (1983a). Null effects of exposure duration and heterogeneity of difference on the same-different disparity in letter matching. Perception \& Psychophysics, 33, 163-171.

Proctor, R. W., \& RAO, K. B. (1983b). Reinstating the original principles of Proctor's unified theory for matching-task phenomena: An evaluation of Krueger and Shapiro's reformulation. Psychological Review, 90, 21-37. 